\title{
Hybrid stars from a constrained equation of state
}

\author{
Márcio Ferreira ${ }^{1, *}$, Renan Câmara Pereira ${ }^{1, * *}$, and Constança Providência ${ }^{1, * * *}$ \\ ${ }^{1}$ CFisUC, Department of Physics, University of Coimbra, P-3004 - 516 Coimbra, Portugal
}

\begin{abstract}
We determine, within a meta-model, the properties of the nuclear matter equation of state (EoS) that allow for a phase transition to deconfinement matter. It is shown that the properties that define the isoscalar channel are the ones that are affected, in particular, a phase transition implies much larger values of the skewness and kurtosis. The effect of multi-quark interaction channels in the description of the quark phase in hybrid stars is also studied. NS properties, such as the mass and radius of the quark core, show an interplay dependence between the 8-quark vector and the 4-quark isovector-vector interactions. We show that low mass NS, $M \sim 1.4 M_{\odot}$, may already contain a quark core, and satisfy all existing NS observational constraints. We discuss the strangeness content of the quark core and its influence on the speed of sound.
\end{abstract}

\section{Introduction}

The physics of neutron stars (NS) can be studied by combining astrophysical observations of electromagnetic radiation, astro-particles and gravitational waves $(\mathrm{GW})$ - multimessenger astrophysics. The recent observation of the massive pulsars, PSR J0348+0432 with $M=2.01 \pm 0.04 M_{\odot}[1]$ and or MSP J0740+6620, a mass $2.08_{-0.07}^{+0.07} M_{\odot}[2,3]$ impose strong constraints on the equation of state $(\mathrm{EoS})$ of nuclear matter. The estimation of an upper bond on tidal deformability of a NS star by the LIGO/Virgo collaboration from the GW170817 event is important in further constraining the high density behavior of the NS EoS $[4,5]$. While a not too stiff EoS is favored from the upper bond on the tidal deformability, massive pulsars require, on the other hand, a considerably stiff EoS for the NS. Such apparent tension might indicate the presence of exotic matter in NS, i.e., non-nucleonic degrees of freedom. The transition, via a first-order phase transition, from hadronic to quark matter at high densities could be an explanation for the existence of low tidal deformabilities for NS masses around $1.4 M_{\odot}$. There is a great interest in finding observational signatures sensible to the presence of exotic matter inside NS [6-8].

Herein, hybrid NS are described by applying a two-model approach: we choose an hadronic model for the confined phase and a quark model for the quark phase, which are connected through a first-order phase transition (applying the Maxwell construction). The quark model chosen is the well known Nambu-Jona-Lasinio (NJL) model, an effective model of QCD, that considers chiral symmetry preserving interactions $[9,10]$. The use of the NJL model in such two-model approaches has been widely explored both considering a firstorder phase transition [11-16] and with a smooth (crossover) transition between both phases

\footnotetext{
*e-mail: marcio.ferreira@uc.pt

**e-mail: renan.pereira@student.uc.pt

***e-mail: cp@ fis.uc.pt
} 
$[17,18]$. Starting from the three-flavor NJL model, we study the effect of several interaction channels in the existence and stability of hybrid stars, and specifically on quark core properties [19-21].

\section{Equation of state of hybrid NS}

The matter inside hybrid stars is modelled via a two-model approach: an hadronic phase is connected to a quark phase through a first-order phase transition, i.e., a Maxwell construction is implemented. Therefore, we need to choose both an hadronic model and a quark model.

\subsection{Hadronic EoS}

In the following, we consider two types of hadronic EoS: a) a set of metamodels based on a Taylor expansion around saturation density [22]; b) a relativistic mean-field model with density dependent couplings DDME2 [23], which satisfies well accepted nuclear matter properties at saturation $\left(K_{\text {sat }}=250 \mathrm{MeV}, E_{\text {sym }}=32.3 \mathrm{MeV}, L_{\text {sym }}=51.2 \mathrm{MeV}, K_{\text {sym }}=-87 \mathrm{MeV}\right)$, and besides describes two solar mass stars.

In the following, we summarize the metamodel considered. The energy per particle functional of homogeneous nuclear matter, $\mathcal{E}$, can be written as:

$$
\mathcal{E}\left(n_{n}, n_{p}\right)=e_{\text {sat }}(n)+e_{\text {sym }}(n) \delta^{2} .
$$

where $n_{n}$ and $n_{p}$ are neutron and proton densities, $n=n_{n}+n_{p}$ is the baryonic density and $\delta=\left(n_{n}-n_{p}\right) / n$ is the asymmetry. Following previous studies, e.g. [22], we parametrize the hadronic EoS as a Taylor expansion around the saturation density. We consider a fourth order expansion around saturation density, $n_{0}$ :

$$
e_{\text {sat }}(x)=\sum_{n=0}^{4} \frac{1}{n !} P_{I S}^{(n)} x^{n}, \quad e_{\text {sym }}(x)=\sum_{n=0}^{4} \frac{1}{n !} P_{I V}^{(n)} x^{n}
$$

where, $x=\frac{\left(n-n_{0}\right)}{3 n_{0}}$. The coefficients of $e_{s a t}(n)$ and $e_{s y m}(n)$ can be identified, respectively, with the isoscalar and isovector empirical parameters,

$$
P_{I S}^{(k)}=\left.\left(3 n_{0}\right)^{k} \frac{\partial^{k} e_{s a t}}{\partial n^{k}}\right|_{\left\{\delta=0, n=n_{0}\right\}}, \quad P_{I V}^{(k)}=\left.\left(3 n_{0}\right)^{k} \frac{\partial^{k} e_{s y m}}{\partial n^{k}}\right|_{\left\{\delta=0, n=n_{0}\right\}} .
$$

The correspondence between the coefficients and the empirical parameters can then be written as: $E_{\text {sat }}, K_{\text {sat }}, Q_{\text {sat }}, Z_{\text {sat }} \rightarrow P_{I S}^{(0)}, P_{I S}^{(2)}, P_{I S}^{(3)}, P_{I S}^{(4)}$, and $E_{\text {sym }}, L_{\text {sym }}, K_{\text {sym }}, Q_{\text {sym }}, Z_{\text {sym }} \rightarrow$ $P_{I V}^{(0)}, P_{I V}^{(1)}, P_{I V}^{(2)}, P_{I V}^{(3)}, P_{I V}^{(4)}$.

The advantages of taking a meta-model for the hadron phase can be summarized in the following two points: (i) its flexibility to build an hybrid EoS with $1.97 M_{\odot}$ maximum mass; (ii) the possibility of choosing the empirical nuclear parameters that satisfy uncertainties on the EoS parameters known from experiments and other theoretical studies. From all the metamodel EoSs built, 354 of them allow for a phase transition to quark matter. In Table 1, the mean and standard deviation of the properties of the both sets of EoS, nucleonic and hybrid, are given. We also include a third set of EoS that allows for a phase transition to quark matter at a quite low baryonic density, i.e. the transition density $n_{t}$ satisfies $1.3<n_{t} / n_{0}<2.5$. Comparing the three meta-models sets we conclude that a phase transition to quark matter requires larger values of isoscalar properties, in particular, $Q_{\text {sat }}$ and $Z_{s a t}$, and a wider spread, while the isovector channel is essentially not affected. 
Table 1. The mean $\bar{P}_{i}$ and standard deviation $\sqrt{\sigma_{P_{i}}}$ of the multivariate Gaussian, where $\sigma_{P_{i}}$ is the variance of the parameter $P_{i}$. Our EoSs are sampled using the initial distribution for $P_{i}$ assuming that there are no correlations among the parameters. All the quantities are in units of MeV. The values of $E_{\text {sat }}$ and $n_{0}$ are fixed to $-15.8 \mathrm{MeV}$ and $0.155 \mathrm{fm}^{-3}$, respectively. The parameters are given for three

scenarios always considering that a two solar mass star is described by the models: (i) purely nucleonic EoS; (ii) hybrid EoS, all without furhter filters; (iii) hybrid stars with a phase transition in the range $1.3 n_{0}-2.5 n_{0}$.

\begin{tabular}{ccccccccc}
\hline (i) Nucleonic & $K_{\text {sat }}$ & $Q_{\text {sat }}$ & $Z_{\text {sat }}$ & $E_{\text {sym }}$ & $L_{\text {sym }}$ & $K_{\text {sym }}$ & $Q_{\text {sym }}$ & $Z_{\text {sym }}$ \\
\hline mean & 233.95 & 58.62 & -181.97 & 33.32 & 51.56 & -43.96 & 238.21 & 371.91 \\
std & 18.75 & 123.33 & 143.04 & 1.89 & 11.83 & 63.02 & 300.33 & 698.56 \\
\hline (ii) Hybrid (all) & $K_{\text {sat }}$ & $Q_{\text {sat }}$ & $Z_{\text {sat }}$ & $E_{\text {sym }}$ & $L_{\text {sym }}$ & $K_{\text {sym }}$ & $Q_{\text {sym }}$ & $Z_{\text {sym }}$ \\
\hline mean & 236.52 & 241.38 & 362.11 & 33.17 & 49.99 & -37.48 & 191.54 & 503.39 \\
std & 17.73 & 242.03 & 593.75 & 1.84 & 12.55 & 67.23 & 310.48 & 734.69 \\
\hline (iii) Hybrid & $K_{\text {sat }}$ & $Q_{\text {sat }}$ & $Z_{\text {sat }}$ & $E_{\text {sym }}$ & $L_{\text {sym }}$ & $K_{\text {sym }}$ & $Q_{\text {sym }}$ & $Z_{\text {sym }}$ \\
$\left(1.3<n_{t} / n_{0}<2.5\right)$ & & & & & & & & \\
\hline mean & 244.55 & 434.37 & 557.83 & 33.38 & 49.90 & -18.07 & 195.39 & 400.46 \\
std & 16.91 & 174.6 & 743.94 & 2.00 & 11.55 & 57.47 & 300.13 & 672.24 \\
\hline
\end{tabular}

To build an hadronic EoS, we use random sampling to choose a point in the 8-dimensional space of parameters from a multivariate Gaussian with zero covariance:

$$
\operatorname{EoS}_{i}=\left(E_{\text {sym }}, L_{\text {sym }}, K_{\text {sat }}, K_{\text {sym }}, Q_{\text {sat }}, Q_{\text {sym }}, Z_{\text {sat }}, Z_{\text {sym }}\right)_{i} \sim N(\boldsymbol{\mu}, \boldsymbol{\Sigma})
$$

with the mean defined by $\boldsymbol{\mu}^{T}=\left(\bar{E}_{\text {sym }}, \bar{L}_{\text {sym }}, \bar{K}_{\text {sat }}, \bar{K}_{\text {sym }}, \bar{Q}_{s a t}, \bar{Q}_{s y m}, \bar{Z}_{s a t}, \bar{Z}_{\text {sym }}\right)$, and the covariance matrix given by $\Sigma=\operatorname{diag}\left(\sigma_{E_{s y m}}, \sigma_{L_{s y m}}, \sigma_{K_{s a t}}, \sigma_{K_{s y m}}, \sigma_{Q_{s a t}}, \sigma_{Q_{s y m}}, \sigma_{Z_{s a t}}, \sigma_{Z_{s y m}}\right)$.

\subsection{Quark EoS}

For the quark matter phase, we use a SU(3) NJL-type model, given by the following multiquark interaction Lagrangian density:

$$
\begin{aligned}
\mathcal{L} & =\bar{\psi}\left(i \partial-\hat{m}+\hat{\mu} \gamma^{0}\right) \psi+G_{S} \sum_{a=0}^{8}\left[\left(\bar{\psi} \lambda^{a} \psi\right)^{2}+\left(\bar{\psi} i \gamma^{5} \lambda^{a} \psi\right)^{2}\right]-G_{D}\left[\operatorname{det}\left(\bar{\psi}\left(1+\gamma_{5}\right) \psi\right)+\operatorname{det}\left(\bar{\psi}\left(1-\gamma_{5}\right) \psi\right)\right] \\
& -G_{\omega}\left[\left(\bar{\psi} \gamma^{\mu} \lambda^{0} \psi\right)^{2}+\left(\bar{\psi} \gamma^{\mu} \gamma_{5} \lambda^{0} \psi\right)^{2}\right]-G_{\rho} \sum_{a=1}^{8}\left[\left(\bar{\psi} \gamma^{\mu} \lambda^{a} \psi\right)^{2}+\left(\bar{\psi} \gamma^{\mu} \gamma_{5} \lambda^{a} \psi\right)^{2}\right] \\
& -G_{\omega \omega}\left[\left(\bar{\psi} \gamma^{\mu} \lambda^{0} \psi\right)^{2}+\left(\bar{\psi} \gamma^{\mu} \gamma_{5} \lambda^{0} \psi\right)^{2}\right]^{2} \\
& -G_{\rho \rho} \sum_{a=1}^{8} \sum_{b=1}^{8}\left[\left(\bar{\psi} \gamma^{\mu} \lambda^{a} \psi\right)^{2}+\left(\bar{\psi} \gamma^{\mu} \gamma_{5} \lambda^{a} \psi\right)^{2}\right]\left[\left(\bar{\psi} \gamma^{\mu} \lambda^{b} \psi\right)^{2}+\left(\bar{\psi} \gamma^{\mu} \gamma_{5} \lambda^{b} \psi\right)^{2}\right] \\
& -G_{\omega \rho}\left[\left(\bar{\psi} \gamma^{\mu} \lambda^{0} \psi\right)^{2}+\left(\bar{\psi} \gamma^{\mu} \gamma_{5} \lambda^{0} \psi\right)^{2}\right] \sum_{a=1}^{8}\left[\left(\bar{\psi} \gamma^{\mu} \lambda^{a} \psi\right)^{2}+\left(\bar{\psi} \gamma^{\mu} \gamma_{5} \lambda^{a} \psi\right)^{2}\right]
\end{aligned}
$$

The parameters of the quark model, $\hat{m}=\operatorname{diag}\left(m_{u}, m_{d}, m_{s}\right), G_{S}, G_{D}$, and $\Lambda$, are fit to reproduce the vacuum meson masses and decay constants (Table 2), while the remaining parameters are left as free.

We have analyzed the effect of some of these specific channels on hybrid stars in [19-21], where a detailed description can be found. In particular, in the following we discuss the effect of parameters $G_{\omega}, G_{\rho}$ and $G_{\omega \omega}$. The parameters $G_{\rho \rho}$ and $G_{\omega \rho}$ have only a residual or small 
Table 2. Parameters of the quark model: $\Lambda$ is the model cutoff, $m_{u, d, s}$ are the quark current masses, $G_{S}$ and $G_{D}$ are coupling constants. $M_{u, d}$ and $M_{s}$ are the resulting constituent quark masses in the vacuum.

\begin{tabular}{ccccccc}
\hline \hline $\begin{array}{c}\Lambda \\
{[\mathrm{MeV}]}\end{array}$ & $\begin{array}{c}m_{u, d} \\
{[\mathrm{MeV}]}\end{array}$ & $\begin{array}{c}m_{s} \\
{[\mathrm{MeV}]}\end{array}$ & $G_{S} \Lambda^{2}$ & $G_{D} \Lambda^{5}$ & $\begin{array}{c}M_{u, d} \\
{[\mathrm{MeV}]}\end{array}$ & $\begin{array}{c}M_{s} \\
{[\mathrm{MeV}]}\end{array}$ \\
\hline \hline 623.58 & 5.70 & 136.60 & 1.67 & 13.67 & 332.2 & 510.7 \\
\hline
\end{tabular}

effect on the EoS and NS properties. In the following, we will characterize each quark model by the coupling ratios: $\chi_{\omega}=G_{\omega} / G_{S}, \chi_{\omega \omega}=G_{\omega \omega} / G_{S}^{4}, \chi_{\rho}=G_{\rho} / G_{S}, \chi_{\rho \rho}=G_{\rho \rho} / G_{S}^{4}$, and $\chi_{\omega \rho}=G_{\omega \rho} / G_{S}^{4}$.

The thermodynamical potential is determined using the mean-field approximation, from which we calculate the pressure and energy density for cold quark matter, i.e., at zero temperature. The pressure $P$ and energy density $\epsilon$ are defined up to an extra constant term $B$ (the bag constant), i.e., $P \rightarrow P+B$ and $\epsilon \rightarrow \epsilon-B$. This extra parameter will allow us to control the baryonic density at the hadron-quark phase transition. As referred before, for the hadronic phase, we use the metamodel introduced above and the relativistic mean-field model with density dependent couplings model DDME2 [23]. The first-order phase transition from hadronic $(H)$ to quark $(Q)$ matter is accomplished by imposing the Maxwell construction, i.e., $\mu_{B}^{H}=\mu_{B}^{Q}$ and $P^{H}=P^{Q}$, where $\mu_{B}^{H, Q}$ is the baryon chemical potential of each phase.

\section{Results and discussion}

In the following, we study how the quark matter properties affect the existence of stable hybrid stars. First we analize the effect of the vector-isoscalar parameter $\chi_{\omega}=G_{\omega} / G_{S}$ on the values of the mass and radius, respectively, $M_{t}$ and $R_{t}$, of the star where quarks start to nucleate (see Fig. 1 left panel) and the radius and mass of the quark core, respectively, $M_{Q C}$ and $R_{Q C}$, as a function of the maximum NS mass (Fig. 1 center and right panels). In this figure each hybrid EoS is represented by one point whose color designates a specific value of $\chi_{\omega}$, shown in color scale. The radius and mass of the quark core, $R_{Q C}$ and $M_{Q C}$, is determined from the quark content of the most massive stable NS that contains quark matter. As $\chi_{\omega}$ increases, the appearance of quarks occurs for larger NS masses and radii. Quark cores in massive NS, with $M>2.4 M_{\odot}$, are attainable with $\chi_{\omega}>0.6$. However, the quark core size (center and right panels), of the EoSs that sustain high values of $M_{\max }$, is quite small. The distribution of $M_{Q C}$ has a mean value of $0.08 M_{\odot}$ and a maximum value of $1.29 M_{\odot}$, whereas the mean value of $R_{Q C}$ is $1.99 \mathrm{~km}$ and the maximum value of $8.78 \mathrm{~km}$. Quark cores having radii as large as $7 \mathrm{~km}$ have been also obtained in [24] using a quite different approach.

We next analyze the impact of $\left\{\chi_{\omega \omega}, \chi_{\rho}\right\}$ (with $\chi_{\omega}=0$ ) in the $M(R)$ diagram (Fig. 2). Larger quark branches are realized for larger $\chi_{\omega \omega}$ values, where massive NS $\left(M>2 M_{\odot}\right)$ are already attained for $\chi_{\omega \omega}=15$ and 20. This is explained because the magnitude of the $\chi_{\omega \omega}$ term is still small at the hadron-quark transition, not affecting the transition density. However, at larger densities it contributes with a strong repulsive term, gives rise to a hard quark EoS, and larger star masses are reached.

The NS mass of the quark onset increases with $\chi_{\rho}$ (regardless of $\chi_{\omega \omega}$ ) because the coupling shifts the onset of strange quarks to lower densities, making the quark EoS softer. A second effect of the softening of the EoS is the decrease of the quark branch: the smoothing of the EoS caused by the appearance of strange quarks prevents too massive stars to occur.

We have determined the binary effective tidal deformability $\tilde{\Lambda}$ for $M_{\text {chirp }}=1.186 M_{\odot}$ and $0.73 \leq q=M_{1} / M_{2} \leq 1$ (GW170817 event [26]). Figure 3 shows the $q(\tilde{\Lambda})$ diagram 

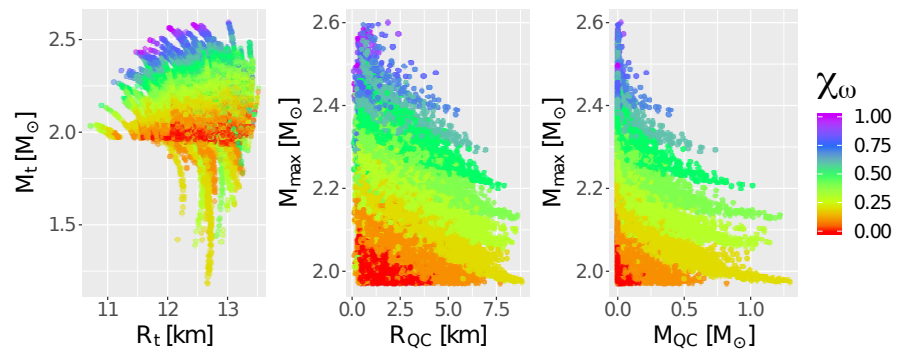

Figure 1. The $M_{t}$ vs $R_{t}$ (left), which corresponds to the $(M, R)$ where the onset of quark matter occurs, and the diagrams of maximum NS mass vs. quark core radius (center) and mass (right) as a function of $\chi_{\omega}$ (color scale) for each hybrid EoS.

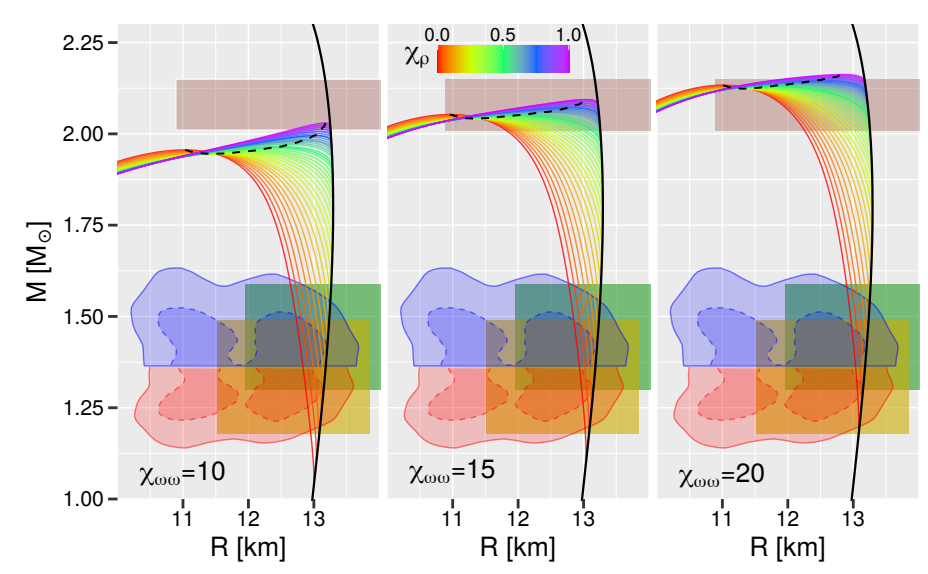

Figure 2. $M(R)$ diagrams as a function of $\chi_{\rho}$ (color scale) for three values of $\chi_{\omega \omega}: 10$ (left), 15 (center), and 20 (right). The solid black line represents the purely hadronic sequence, and the black dashed line indicates the maximum mass of each hybrid EoS. The top blue and bottom red regions indicate, respectively, the $90 \%$ (solid) and 50\% (dashed) credible intervals of the LIGO/Virgo analysis for the GW170817 event [26]. The rectangular regions enclosed by dotted lines indicate the constraints from the millisecond pulsar PSR J0030+0451 NICER x-ray data [28, 29]. The top brown region is from [25].

as function of $\chi_{\omega \omega}$ (color scale) for three $\left\{\chi_{\omega}, \chi_{\rho}\right\}$ sets. The hybrid EoS are concentrated around $\tilde{\Lambda}=650-750$ and fall inside the $90 \%$ credible region for $0.8 \leq q \leq 1$ when $\left(\chi_{\omega}=0, \chi_{\rho}=0\right)$. Highly asymmetric systems, $q<0.8$, have a purely hadronic NS component with low $M$ and large $R$, giving rise to larger $\tilde{\Lambda}$. For the other two sets, $\left(\chi_{\omega}=0.1, \chi_{\rho}=0\right)$ and $\left(\chi_{\omega}=0, \chi_{\rho}=0.1\right)$, (middle and right panels) $\tilde{\Lambda} \sim 800$, and, therefore, they are still compatible with the GW170817 event if moderate asymmetries are considered, i.e., $q>0.8$.

Figure 4 shows the effect of $\left\{\chi_{\omega \omega}, \chi_{\rho}, \chi_{\omega}\right\}$ on various NS properties: the quark core mass $M_{\mathrm{QC}}$, the quark core radius $R_{\mathrm{QC}}$, the speed of sound at the central density $v_{s}^{2}\left(n_{\max }\right)$, and the central density $n_{\max }$. Several conclusions can be drawn: i) while $M_{\mathrm{QC}}$ increases with an increase of $\chi_{\omega \omega}$, the opposite effect occurs for $\chi_{\rho}$ or/and $\chi_{\omega}$, i.e. for larger values of these last two parameters the mass of the quark core reduces a lot. As a result, large quark cores require 


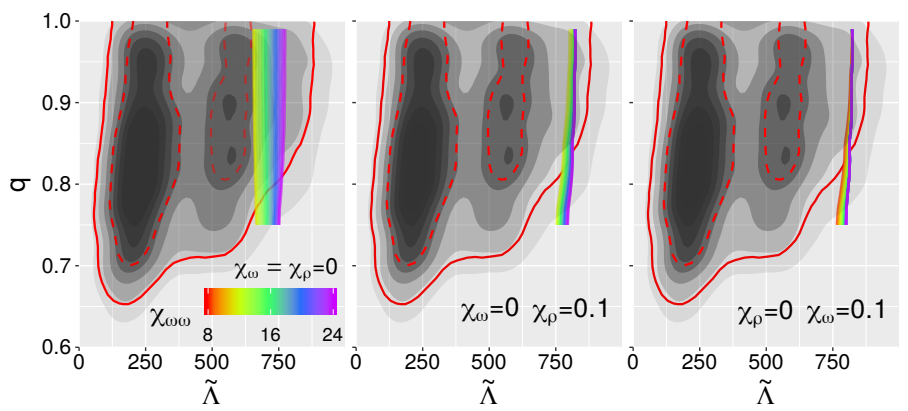

Figure 3. $q(\tilde{\Lambda})$ diagrams for binary systems, with $M_{\text {chirp }}=1.186 M_{\odot}$, as a function of $\chi_{\omega \omega}$ (color scale) for three sets $\left(\chi_{\omega}, \chi_{\rho}\right):(0,0)$ (left), $(0.1,0)$ (center), and $(0,0.1)$ (right). The probability distribution function $P(q, \tilde{\Lambda})$ from the LIGO/Virgo collaboration [27] is displayed in gray gradient, while the red dashed and solid lines indicate the $50 \%$ and $90 \%$ credible regions, respectively.
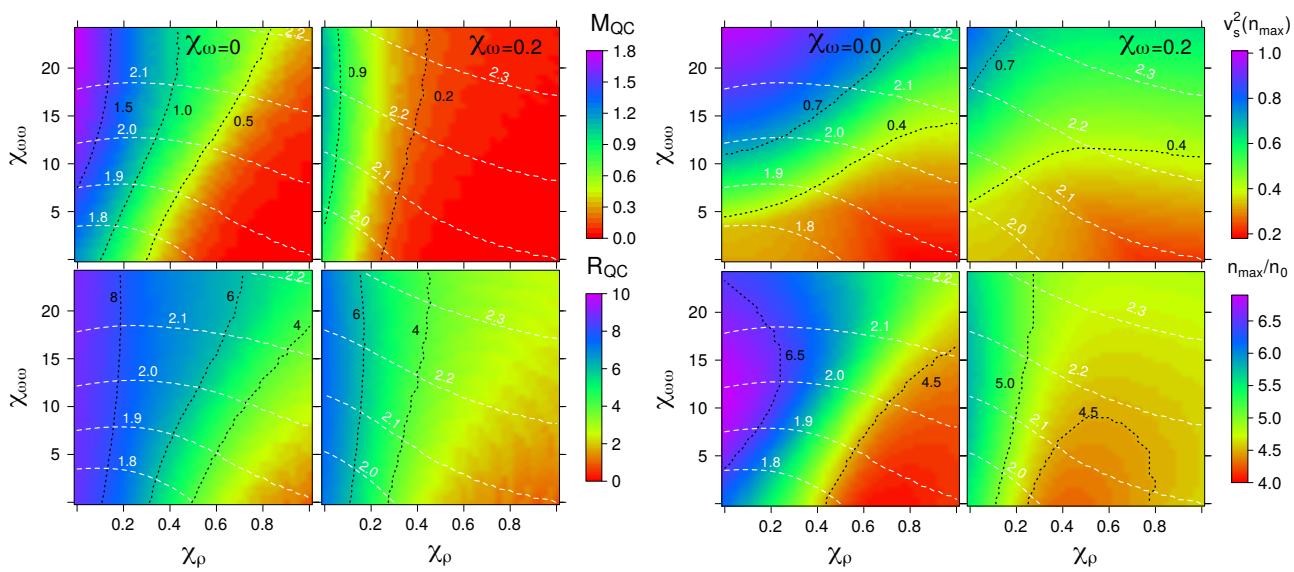

Figure 4. The quark core mass $M_{\mathrm{QC}}\left[M_{\odot}\right]$ (top left), the quark core radius $R_{\mathrm{QC}}[\mathrm{km}]$ (bottom left), the squared speed of sound at the central density $\left[c^{2}\right]$ (top right), and central densities $\left[n_{0}\right]$ of $M_{\max }$ (bottom right) as a function of $\left(\chi_{\omega \omega}, \chi_{\rho}\right)$. The white dashed and black dotted lines represent, respectively, the value of $M_{\max }\left[M_{\odot}\right]$ and specific $\left\{M_{\mathrm{QC}}, R_{\mathrm{QC}}, v_{s}^{2}\left(n_{\max }\right), n_{\max } / n_{0}\right\}$ contour lines.

small values of $\chi_{\rho}$ and $\chi_{\omega}$ and a large $\chi_{\omega \omega}$ coupling; ii) considering $\chi_{\omega}=\chi_{\rho}=0$, massive NS are only realized for $\chi_{\omega \omega} \gtrsim 12$, with very large radii $R_{\mathrm{QC}}$ and speed of sound values $v_{s}^{2}\left(n_{\max }\right)$ close to 1 ; iii) $v_{s}^{2}$ increases quite rapidly with $\chi_{\omega \omega}$ but decreases with $\chi_{\rho}$; iv) the largest central densities occur for $\chi_{\omega}=\chi_{\rho}=0 ; \mathrm{v}$ ) it is possible to have a quark core having a mass of the order of $1 / 3$ of the total star mass, and a speed of sound $v_{s}^{2}\left(n_{\max }\right) \sim 0.6$. The softening of the EoS with a finite $\chi_{\rho}$ due to the earlier onset of strange quarks explains this behavior.

The presence of strangeness inside the star can be understood considering Fig. 5, where the strangeness fraction at the center of the maximum mass star is plotted as a function of $\chi_{\omega \omega}$ and $\chi_{\rho}$ for two values of $\chi_{\omega}, 0$ and 0.1 . The largest fraction of strangeness is attained for $\chi_{\omega}=0, \chi_{\rho}=0.5$ and $\chi_{\omega \omega} \approx 20$. It is interesting to notice that under these conditions the speed of sound squared is of the order of 0.7 and the strangeness fraction of the order of 0.25 . Taking $\chi_{\rho}=0$ the speed of sound square takes values close to 1 and the strangeness fractions 

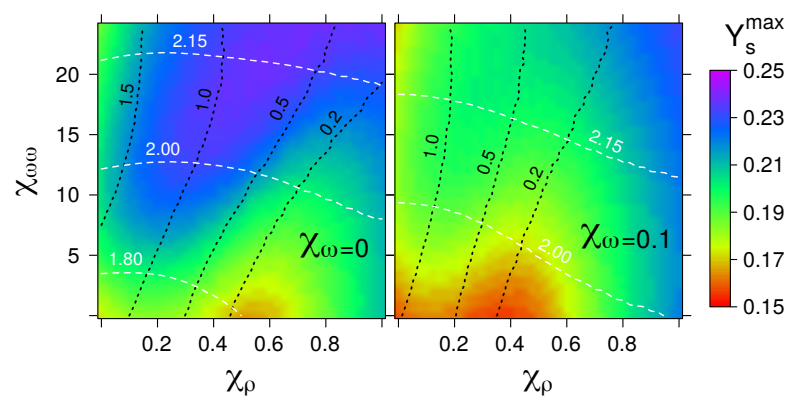

Figure 5. The fraction of $s$-quarks, $Y_{s}^{\max }$, at the center of the maximum mass star, as a function of $\chi_{\omega \omega}$ and $\chi_{\rho}$ for $\chi_{\omega}=0$ (left) and 0.1 (right). The white dashed and black dotted lines represent, respectively, the value of the star maximum mass $M_{\max }\left[M_{\odot}\right]$ and specific $M_{\mathrm{QC}}\left[M_{\odot}\right]$ contour lines.

drops to values of the order of 0.18 . The presence of a larger amount of $s$-quarks prevents the speed of sound to take values close to one, but also reduces the mass of the quark core.

We have studied under which conditions two solar mass hybrid stars may exist. It was shown that a phase transition into a quark phase requires a harder isoscalar channel for the nucleonic EoS, than expected from just considering nucleonic degrees of freedom. We have shown that the present constraints on mass, radius, and tidal deformability from NS observations allow for the existence of hybrid stars with large quark cores. The $\chi_{\omega \omega}$ channel is important in generating massive quark cores, even at moderate quark masses, e.g., $1.4 M_{\odot}$ stars, with $\tilde{\Lambda}<800$. However, The $\chi_{\omega \omega}$ channel may originate very large values for the speed of sound. On the other hand, the $\chi_{\rho}$ channel controls the amount of strangeness inside the star, and reduces the speed of sound.

\section{References}

[1] J. Antoniadis et al., 10.1126/science.1233232 Science 340, 6131 (2013), http://arxiv.org/abs/1304.6875 arXiv:1304.6875 [astro-ph.HE]

[2] H. T. Cromartie, E. Fonseca, S. M. Ransom, P. B. Demorest, Z. Arzoumanian, H. Blumer, P. R. Brook, M. E. DeCesar, T. Dolch, J. A. Ellis, R. D. Ferdman, E. C. Ferrara, N. GarverDaniels, P. A. Gentile, M. L. Jones, M. T. Lam, D. R. Lorimer, R. S. Lynch, M. A. McLaughlin, C. Ng, D. J. Nice, T. T. Pennucci, R. Spiewak, I. H. Stairs, K. Stovall, J. K. Swiggum, and W. W. Zhu, 10.1038/s41550-019-0880-2 Nature Astronomy , 439 (2019), http://arxiv.org/abs/1904.06759 arXiv:1904.06759 [astro-ph.HE]

[3] E. Fonseca et al., Astrophys. J.Lett. 915, L12 (2021), arXiv:2104.00880 [astro-ph.HE]

[4] B. P. Abbott et al. (LIGO Scientific, Virgo), 10.1103/PhysRevLett.119.161101 Phys. Rev. Lett. 119, 161101 (2017), http://arxiv.org/abs/1710.05832 arXiv:1710.05832 [gr-qc]

[5] B. A. et al. (The LIGO Scientific Collaboration and the Virgo Collaboration) (The LIGO Scientific Collaboration and the Virgo Collaboration), 10.1103/PhysRevLett.121.161101 Phys. Rev. Lett. 121, 161101 (2018)

[6] M. G. Alford, S. Han, and K. Schwenzer, 10.1088/1361-6471/ab337a J. Phys. G46, 114001 (2019), http://arxiv.org/abs/1904.05471 arXiv:1904.05471 [nucl-th]

[7] E. R. Most, L. J. Papenfort, V. Dexheimer, M. Hanauske, S. Schramm, H. Stöcker, and L. Rezzolla, 10.1103/PhysRevLett.122.061101 Phys. Rev. Lett. 122, 061101 (2019), http://arxiv.org/abs/1807.03684 arXiv:1807.03684 [astro-ph.HE] 
[8] L. R. Weih, M. Hanauske, and L. Rezzolla, 10.1103/PhysRevLett.124.171103 Phys. Rev. Lett. 124, 171103 (2020), http://arxiv.org/abs/1912.09340 arXiv:1912.09340 [gr-qc]

[9] T. Hatsuda and T. Kunihiro, Phys. Rept. 247 (1994), 221-367 doi:10.1016/03701573(94)90022-1 [arXiv:hep-ph/9401310 [hep-ph]].

[10] M. Buballa, Phys. Rept. 407 (2005), 205-376 doi:10.1016/j.physrep.2004.11.004 [arXiv:hep-ph/0402234 [hep-ph]].

[11] G. Pagliara and J. Schaffner-Bielich, Phys. Rev. D 77 (2008), 063004 doi:10.1103/PhysRevD.77.063004 [arXiv:0711.1119 [astro-ph]].

[12] S. Benic, Eur. Phys. J. A 50 (2014), 111 doi:10.1140/epja/i2014-14111-1 [arXiv:1401.5380 [nucl-th]].

[13] S. Benic, D. Blaschke, D. E. Alvarez-Castillo, T. Fischer and S. Typel, Astron. Astrophys. 577 (2015), A40 doi:10.1051/0004-6361/201425318 [arXiv:1411.2856 [astroph.HE]].

[14] A. Zacchi, M. Hanauske and J. Schaffner-Bielich, Phys. Rev. D 93 (2016) no.6, 065011 doi:10.1103/PhysRevD.93.065011 [arXiv:1510.00180 [nucl-th]].

[15] R. Câmara Pereira, P. Costa and C. Providência, Phys. Rev. D 94 (2016) no.9, 094001 doi:10.1103/PhysRevD.94.094001 [arXiv:1610.06435 [nucl-th]].

[16] X. Wu, A. Ohnishi and H. Shen, Phys. Rev. C 98 (2018) no.6, 065801 doi:10.1103/PhysRevC.98.065801 [arXiv:1806.03760 [nucl-th]].

[17] G. Baym, T. Hatsuda, T. Kojo, P. D. Powell, Y. Song and T. Takatsuka, Rept. Prog. Phys. 81 (2018) no.5, 056902 doi:10.1088/1361-6633/aaae14 [arXiv:1707.04966 [astroph.HE]].

[18] T. Kojo, Universe 4 (2018) no.2, 42 doi:10.3390/universe4020042

[19] M. Ferreira, R. Câmara Pereira and C. Providência, Phys. Rev. D 101 (2020) no.12, 123030 doi:10.1103/PhysRevD.101.123030 [arXiv:2005.10543 [nucl-th]].

[20] M. Ferreira, R. Câmara Pereira and C. Providência, Phys. Rev. D 102 (2020) no.8, 083030 doi:10.1103/PhysRevD.102.083030 [arXiv:2008.12563 [nucl-th]].

[21] M. Ferreira, R. Câmara Pereira and C. Providência, Phys. Rev. D 103 (2021) no.12, 123020 doi:10.1103/PhysRevD.103.123020 [arXiv:2105.06239 [nucl-th]].

[22] J. Margueron, R. H. Casali, and F. Gulminelli, Phys. Rev. C 97 (2018), 025805

[23] G. A. Lalazissis, T. Nikšić, D. Vretenar, and P. Ring, 10.1103/PhysRevC.71.024312 Phys. Rev. C 71, 024312 (2005)

[24] E. Annala, T. Gorda, A. Kurkela, J. Nättilä, and A. Vuorinen, Nat. Phys. 16 (2020), 907.

[25] M. C. Miller, et al., Astrophys. J. Lett. 918 (2021) no.2, L28.

[26] B. P. Abbott et al. [LIGO Scientific and Virgo], Phys. Rev. Lett. 121 (2018) no.16, 161101 doi:10.1103/PhysRevLett.121.161101 [arXiv:1805.11581 [gr-qc]].

[27] B. P. Abbott et al. [LIGO Scientific and Virgo], Phys. Rev. X 9 (2019) no.1, 011001 doi:10.1103/PhysRevX.9.011001 [arXiv:1805.11579 [gr-qc]].

[28] T. E. Riley, A. L. Watts, S. Bogdanov, P. S. Ray, R. M. Ludlam, S. Guillot, Z. Arzoumanian, C. L. Baker, A. V. Bilous and D. Chakrabarty, et al. Astrophys. J. Lett. 887 (2019) no.1, L21 doi:10.3847/2041-8213/ab481c [arXiv:1912.05702 [astro-ph.HE]].

[29] M. C. Miller, F. K. Lamb, A. J. Dittmann, S. Bogdanov, Z. Arzoumanian, K. C. Gendreau, S. Guillot, A. K. Harding, W. C. G. Ho and J. M. Lattimer, et al. Astrophys. J. Lett. 887 (2019) no.1, L24 doi:10.3847/2041-8213/ab50c5 [arXiv:1912.05705 [astro-ph.HE]]. 